> IMGT®, the international ImMunoGeneTics information system ${ }^{\circledR}$, a été créé en 1989 à Montpellier par le Laboratoire d'ImmunoGénétique Moléculaire (LIGM) des professeurs MariePaule et Gérard Lefranc (Université Montpellier 2 et CNRS). IMGT® est spécialisé dans les séquences, structures et données génétiques des immunoglobulines ou anticorps, des récepteurs $T$, du complexe majeur d'histocompatibilité, des protéines des superfamilles IgSF et MhcSF, et des protéines apparentées du système immunitaire. L'analyse des données et la standardisation de leur annotation ont été possibles grâce à la création d'IMGT-ONTOLOGY, la première ontologie dans le domaine. IMGT® est aujourd'hui le système de référence international en immunogénétique et immunoinformatique, utilisé en recherche fondamentale, médicale et biotechnologique, et en particulier, en ingénierie et humanisation des anticorps. <

\section{IMGT ${ }^{\circledR}$, vingt ans déjà !}

IMGT®, the international ImMunoGeneTics information system ${ }^{\circledR 1}$ [1] fête cette année son vingtième anniversaire. Créé en 1989 à Montpellier par le Laboratoire d'ImmunoGénétique Moléculaire (LIGM) des professeurs Marie-Paule et Gérard Lefranc (Université Montpellier 2 et CNRS), IMGT® est le premier et actuellement le seul système d'information intégré en immunogénétique et immunoinformatique. IMGT®, marque déposée du CNRS (France, Union européenne, Canada et États-Unis), est spécialisé dans les séquences, structures et données génétiques des immunoglobulines (IG) ou anticorps, des récepteurs des lymphocytes T (TR), du complexe majeur d'histocompatibilité (MHC, major histocompatibility complex en anglais), des protéines des superfamilles IgSF (immunoglobulin superfamily) et MhcSF (Mhc super-

${ }^{1}$ http://www.imgt.org
Université Montpellier 2, IMGT, LIGM, Institut

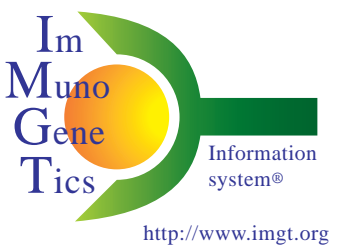
de génétique humaine, UPR CNRS 1142, 141, rue de la Cardonille, 34396 Montpellier cedex 5 , France.

Marie-Paule.Lefranc@igh.cnrs.fr http://www.imgt.org

family), et des protéines apparentées du système immunitaire. IMGT ${ }^{\circledR}$ est composé de plusieurs bases de données (quatre de séquences, une de gènes, une de structures 3D) et d'une quinzaine d'outils interactifs (Figure 1) et de plus de 10000 pages de ressources Web. En raison de la complexité de leur synthèse et de leur incroyable diversité, les molécules du système immunitaire, et en particulier les anticorps, doivent être traitées d'une façon spécifique. L'analyse des données et la standardisation de leur annotation ont été possibles grâce à la création d'IMGT-ONTOLOGY, la première ontologie dans le domaine $[2,3]$. IMGT® est aujourd'hui le système de référence international en immunogénétique et immunoinformatique, utilisé en recherche fondamentale, médicale et biotechnologique.

Un organisme humain synthétise plus de $10^{12}$ anticorps et plus de $10^{12}$ récepteurs $T$ différents pour faire face aux agressions extérieures de la part des virus, bactéries et parasites et pour lutter contre ses propres cellules malignes. C'est par des mécanismes complexes de réarrangements de I'ADN et, dans le cas des gènes IG, de mutations somatiques, qu'un nombre aussi important de récepteurs d'antigènes peut être obtenu $(\rightarrow) . \quad(\rightarrow)$ voir M. Fougereau, Créé en 1989 pour gérer cette page 997 complexité des gènes, séquences et structures des IG et TR, IMGT® est aujourd'hui une source unique de 


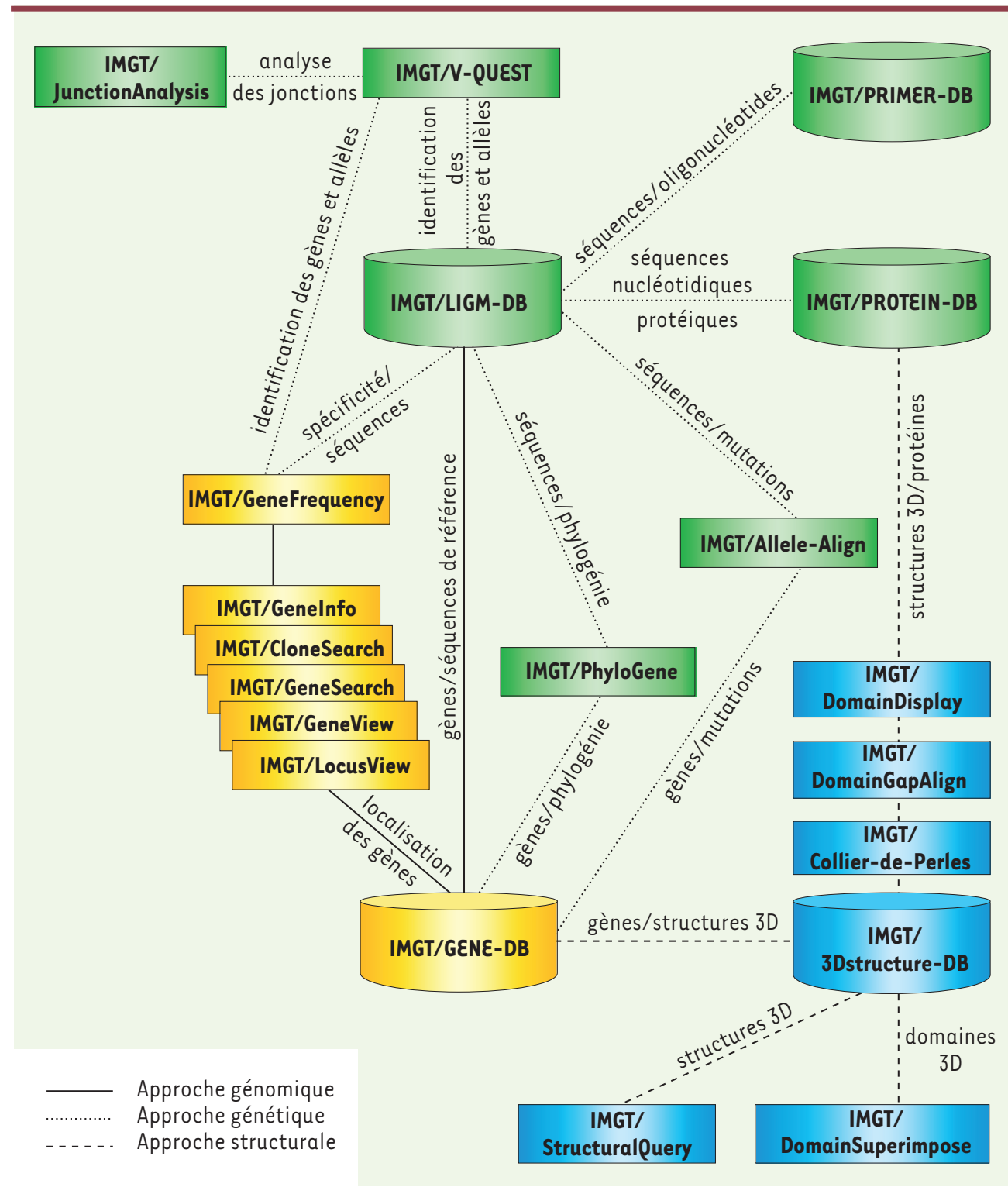

Figure 1. IMGT ${ }^{\circledR}$, the international ImMunoGeneTics information system ${ }^{\circledR}$. IMGT ${ }^{\circledR}$ (http://www.imgt.org) comprend des bases de données et des outils d'analyse (respectivement représentés sous forme de cylindres et de rectangles) et plus de 10000 pages de ressources Web [1]. Les séquences et les gènes, ainsi que les structures tridimensionnelles, sont analysés et gérés selon les trois approches biologiques: génétique, génomique et structurale.

les espèces domestiques et sauvages), recherche génomique (étude de la diversité et de l'évolution des gènes de la réponse immunitaire adaptative), recherche en biologie structurale (évolution des domaines des protéines des superfamilles IgSF et MhcSF) et dans les biotechnologies relatives aux projets de l'Human proteome organisation (HUPO) et à l'ingénierie des anticorps (single chain Fragment variable

connaissances en immunogénétique au niveau international. II n’y a pas d'équivalent en Europe, aux États-Unis, au Japon et nulle part ailleurs dans le monde. Le serveur Web d'IMGT® à Montpellier reçoit des accès de plus de 80000 sites différents par an, avec une moyenne de plus de 150000 requêtes par mois. Les utilisateurs sont répartis à parts égales entre l'Europe, les États-Unis et le reste du monde. IMGT® est reconnu pour la richesse et la qualité de ses données scientifiques et son interface conviviale.

IMGT® est utilisé par des chercheurs d'équipes académiques et industrielles dans de multiples domaines de recherche: recherche fondamentale, recherche médicale (analyse des répertoires des anticorps et des sites de reconnaissance des récepteurs $T$ dans les réponses immunitaires normales contre les agents infectieux et les cellules malignes, et lors des réponses anormales au cours de maladies autoimmunes et de syndromes lymphoprolifératifs, leucémies, lymphomes, myélomes), recherche vétérinaire (répertoire des IG et TR dans
scFv, banques combinatoires, phage display, anticorps chimériques, humanisés et humains) $(\rightarrow)$, recherche pour le diagnostic, le pronostic et le suivi thérapeutique des leucémies, lymphomes et myélomes (identification du ou des clone(s) malin(s) et évaluation de $\rightarrow$ voir A. Beck et al., page 1024 ; R. Abès et al., page 1011 ; M. Cogné et al. page 1149 la maladie résiduelle) et dans les approches thérapeutiques (greffes, immunothérapie, vaccinologie). IMGT est accessible à l'adresse suivante: http://www.imgt. org, gratuitement pour la recherche académique, mais par contrats et licences pour les sociétés comme Centocor Inc., Johnson and Johnson (États-Unis), Merck \& Co Inc. (États-Unis), Amgen Inc. (États-Unis), SanofiAventis GmbH (Allemagne), Chugai Pharmaceutical Co., Ltd (Japon), etc. 
DES SÉPUENCES AUX STRUCTURES 3D

CAMPATH-1H|1bey_H|VH-CHI|219 AA|23397.2 $\mathrm{MW} \mid \mathrm{VH}+\mathrm{CH} \mathrm{I}$

QVQLDESGPGLVRPSPTLSLTCTVSGFTFTDFYMNWVRQ PPGRGLEWIGFIRDKAKGYTT

EYNPSVKGRVTMLVDTSKNOFSLRLSSVTAADTAVYYCAR EGHTAAPFDYWGQGSLVTVS

SASTKGPSVFPLAPSSKSTSGGTAALGCLVKDYFPEPVTVS WNSGALTSGVHTFPAVLPS

SGLYSLSSVVTVPSSSLGTPTYICNVNHKPSSNTKVDKKV
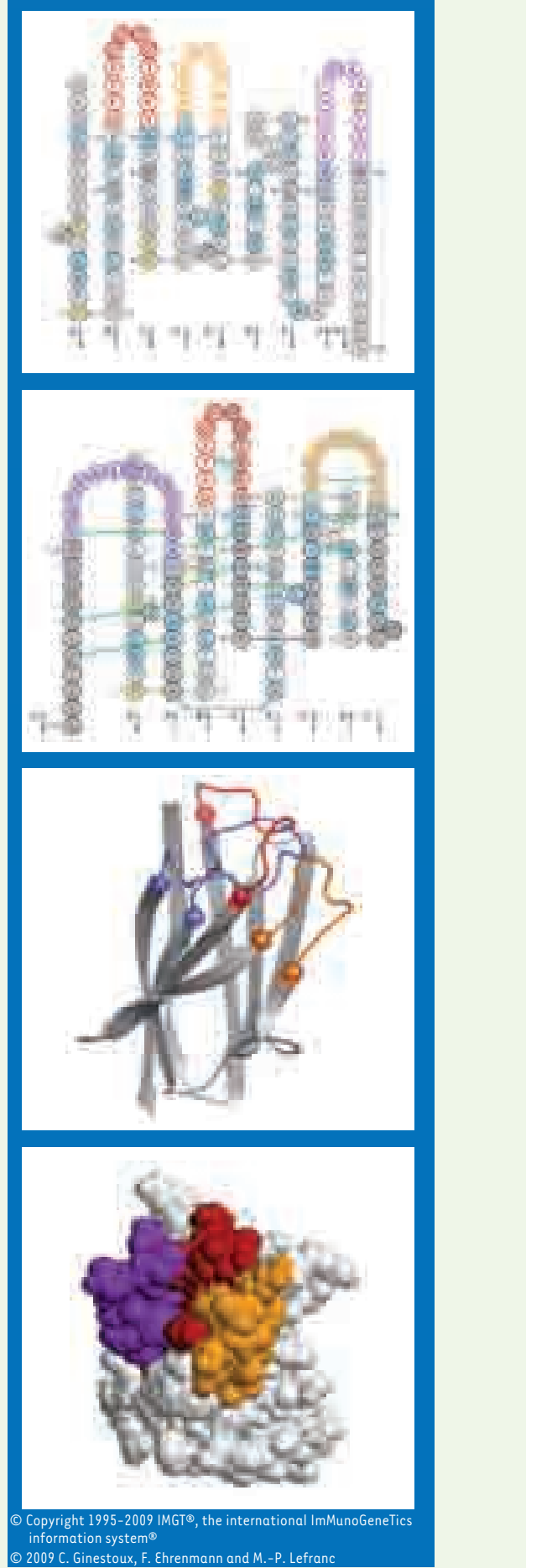

Figure 2. Des séquences aux structures 3D. La numérotation unique IMGT permet de représenter, de manière standardisée, la séquence en acides aminés d'un domaine variable d'anticorps sous forme d'un IMGT Collier de Perles, sur un plan ou deux plans. Les trois boucles hypervariables ou complementarity determining regions (CDR) sont visualisées: CDR1-IMGT (rouge), CDR2-IMGT (orange) et CDR3-IMGT (violet). Ce sont ces CDR qui confèrent la spécificité d'un anticorps et sont greffés lors de l'humanisation d'un anticorps.

\section{IMGT ${ }^{\circledR}$, système de référence international}

IMGT ${ }^{\circledR}$ est devenu le véritable système de référence pour la nomenclature des gènes des immunoglobulines et des récepteurs $T[4,5]$. Ce sont les noms des gènes IG et TR définis par IMGT ${ }^{\circledR}$ qui sont approuvés par le Human genome organisation (HUGO) nomenclature committee (HGNC) et utilisés par les laboratoires du monde entier [6]. La base de gènes Entrez Gene du National center for biotechnology information (NCBI) aux États-Unis fait des liens directs sur les entrées de la base de gènes d'IMGT ${ }^{\circledR}$, IMGT/GENE-DB [7].

La base de données de séquences nucléotidiques d'IMGT®, IMGT/LIGMDB [8], et les outils d'analyse de séquences, IMGT/V-QUEST [9] et IMGT/JunctionAnalysis [10], ont rapidement été adoptés par les laboratoires de recherche, pharmaceutiques et hospitaliers pour l'étude des répertoires. À titre d'exemple, l'European research initiative on CLL (chronic lymphocytic leukemia) (ERIC) qui regroupe dans 26 pays 130 laboratoires travaillant sur les leucémies lymphoïdes chroniques (LLC ou CLL en anglais) a recommandé I'utilisation d'IMGT/V-QUEST comme référence pour la détermination des mutations des gènes IGHV (gènes variables $V$ du locus des chaînes lourdes $H$ des IG) des patients atteints de LLC [11]. Les résultats standardisés d'IMGT/V-QUEST sont cruciaux car le taux de mutations est un facteur pronostic important des LLC: le pronostic est favorable si les gènes sont mutés et défavorable si les gènes ne sont pas mutés.

La base de structures 3D d'IMGT® ${ }^{\circledR}$ IMGT/3Dstructure-DB [12], et les outils d'analyse de structures, IMGT/DomainGapAlign, IMGT/DomainSuperimpose, intègrent la numérotation unique IMGT ${ }^{\circledR}$ et la représentation graphique «IMGT Collier de Perles» (Figure 2) qui permet de passer facilement d'une séquence protéique à une structure 3D [13]. Dans le cas d'un domaine variable d'anticorps, les trois boucles hypervariables qui assurent la spécificité de l'anticorps vis-à-vis de l'antigène sont aisément visualisées. Aujourd'hui les IMGT Collier de Perles ont été adoptés par toute la communauté scientifique, et l'expression est en français dans les publications scientifiques. Ces résultats sont particulièrement importants dans le domaine de l'ingénierie et de I'humanisation des anticorps thérapeutiques [14, 15]. Lorsqu'un anticorps murin est injecté à un patient, une réponse immunitaire se produit contre cet anticorps d'une autre espèce, ce qui réduit son efficacité. L'humanisation consiste à greffer les boucles hypervariables murines, qui confèrent la spécificité, sur un anticorps humain. L'outil IMGT/Collier-de-Perles permet de délimiter précisément les parties à greffer. Ce qui explique l'intérêt des industries pharmaceutiques pour les bases et outils d'IMGT$^{\circledR}$. $\diamond$ 


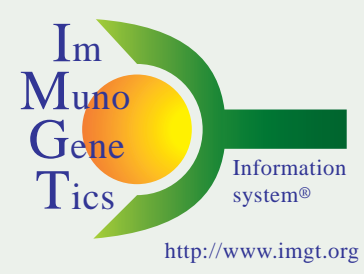

\section{IMGT ${ }^{\circledR}$}

IMGT ${ }^{\circledR}$ est membre institutionnel académique de l'International Medical Informatics Association (IMIA) partenaire du projet européen ELIXIR. Plate-forme RIO depuis 2001. Plate-forme IBiSA infrastructures biologie santé et agronomie depuis 2007. Membre du Réseau national des plates-formes bioinformatiques ( $\mathrm{ReNaBi}$ ). Grand plateau technique pour la recherche (GPTR) région Languedoc-Roussillon depuis 2005. Plan pluri-formation de I'Université Montpellier 2 depuis 1999. Plateforme bioinformatique de I'IFR3 et du Cancéropôle Grand Sud-Ouest (GSO).

\section{SUMMARY}

Antibody databases: IMGT ${ }^{\circledR}$, a French platform of world-wide interest

IMGT ${ }^{\circledR}$, the international ImMunoGeneTics information system ${ }^{\circledR}$, was created in 1989 in Montpellier by the Laboratoire d'ImmunoGénétique Moléculaire (LIGM) headed by the Professors Marie-Paule and Gérard Lefranc (University Montpellier 2 and CNRS). IMGT® is specialized in the sequences, structures and genetic data of the immunoglobulins (IG) or antibodies, T cell receptors (TR), major histocompatibilité complex (MHC), proteins of the IgSF and MhcSF superfamilies, and related proteins of the immune system. The data analysis and the standardization of the annotation are based on IMGT-ONTOLOGY, the first ontology in the domain. IMGT ${ }^{\circledR}$ is to-day the international reference system in immunogenetics and immunoinformatics, used in fundamental, medical and biotechnological research and, in particular, in antibody engineering and humanization. $\diamond$

\section{CONFLIT D'INTÉRÊTS}

L'auteur déclare n'avoir aucun conflit d'intérêts concernant les données publiées dans cet article.

\section{RÉFÉRENCES}

1. Lefranc MP, Giudicelli V, Ginestoux C, et al. IMGT ${ }^{\circledR}$, the international ImMunoGeneTics information system ${ }^{\circledR}$. Nucleic Acids Res 2009 ; 37 : D1006-12.

2. Giudicelli V, Lefranc MP. Ontology for Immunogenetics: IMGT-ONTOLOGY. Bioinformatics $1999 ; 15$ : 1047-54.

3. Duroux P, Kaas $Q$, Brochet $X$, et al. IMGT-Kaleidoscope, the Formal IMGT-ONTOLOGY paradigm. Biochimie 2008 ; $90: 570-83$.

4. Lefranc MP, Lefranc G. The immunoglobulin FactsBook. London: Academic Press, $2001: 458 \mathrm{p}$.

5. Lefranc MP, Lefranc G. The T cell receptor FactsBook. London: Academic Press, $2001: 398 \mathrm{p}$.

6. Lefranc MP. WHO-IUIS Nomenclature subcommittee for immunoglobulins and T cell receptors. Dev Comp Immunol 2008 ; 32 : 461-3.

7. Giudicelli V, Chaume D, Lefranc MP. IMGT/GENE-DB: a comprehensive database for human and mouse immunoglobulin and T cell receptor genes. Nucleic Acids Res 2005 ; 33 : D256-61.

8. Giudicelli V, Ginestoux C, Folch G, et al. IMGT/LIGM-DB, the IMGT® comprehensive database of immunoglobulin and T cell receptor nucleotide sequences. Nucleic Acids Res 2006 ; 34 : D781-4.

9. Brochet X, Lefranc MP, Giudicelli V. IMGT/V-QUEST: the highly customized and integrated system for IG and TR standardized V-J and

V-D-J sequence analysis. Nucleic Acids Res 2008 ; 36 : W503-8.

10. Yousfi Monod M, Giudicelli V, Chaume D, Lefranc MP. IMGT/ JunctionAnalysis : the first tool for the analysis of the immunoglobulin and T cell receptor complex V-J and V-D-J junctions. Bioinformatics $2004 ; 20: i 379-85$.

11. Ghia P, Stamatopoulos K, Belessi C, et al. On behalf of the European research Initiative on CLL (ERIC). ERIC recommendations on IGHV gene mutational status analysis in chronic lymphocytic leukaemia. Leukemia $2007 ; 21: 1-3$.

12. Kaas $Q$, Ruiz M, Lefranc MP. IMGT/3Dstructure-DB and IMGT/ StructuralQuery, a database and a tool for immunoglobulin, T cell receptor and MHC structural data. Nucleic Acids Res 2004 ; 32 : D208-10.

13. Kaas $Q$, Lefranc MP. IMGT Colliers de Perles: standardized sequencestructure representations of the IgSF and MhcSF superfamily domains. Curr Bioinformatics $2007 ; 2: 21-30$.

14. Magdelaine-Beuzelin C, Kaas $Q$, Wehbi V, et al. Structure-function relationships of the variable domains of monoclonal antibodies approved for cancer treatment. Crit Rev Oncol Hematol 2007 ; 64: 210-25.

15. Pelat T, Bedouelle H, Rees AR, et al. Germline humanization of a nonhuman Primate antibody that neutralizes the anthrax toxin, by in vitro and in silico engineering. J Mol Biol 2008; $384: 1400-7$.

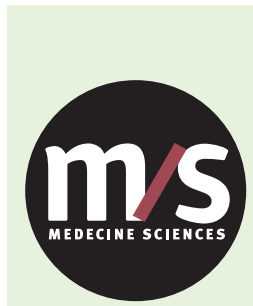

$>$ Grâce à $m / s$, vous vivez en direct

Tarifs d'abonnement M/S - 2010 les progrès des sciences biologiques et médicales

Abonnez-vous

à Médecine/Sciences
Bulletin d'abonnement page VI dans ce numéro de $\mathrm{m} / \mathrm{s}$
TIRÉS À PART

M.P. Lefranc 